\title{
Overview of problems in large-scale wind integrations
}

\author{
Xiaoming YUAN (ه)
}

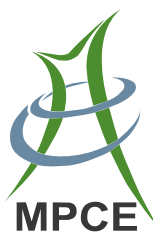

\begin{abstract}
Wind power has been developing rapidly in major countries in the past 10 years. The distinct static and dynamic characteristics of output power compared with conventional generations pose significant challenges on power system adequacy and stability and constraints on the penetration level of wind power in power systems. Based on the uniqueness of wind power versus conventional generations, we discuss its implications on power system adequacy and stability and propose basic solutions for facilitating largescale integrations of wind power into the power system.
\end{abstract}

Keywords Wind power, Adequacy, Stability, Variability and uncertainty, Disturbance mitigating and disturbance ride through

\section{Introduction}

Wind power installation has been growing at a rapid pace over the last decade in most countries and regions due to technology maturity and cost competitiveness of turbines among other technologies. Targets of Europe and the US in 2007 for wind installation will meet $20 \%$ of their electricity consumptions by 2030, reaching 180 and $305 \mathrm{GW}$, respectively $[1,2]$. The "Wind Power Development Roadmap" proposed jointly by the National Development and Reform Committee (NDRC) of China and the International Energy Agency (IEA) also set forth wind installation targets for China to reach 200, 400, and 1,000 GW by 2020, 2030, and

Received: 30 July 2012 / Accepted: 8 October 2012 / Published online: 11 July 2013

(C) The Author(s) 2013. This article is published with open access at Springerlink.com

X. YUAN, College of Electrical and Electronic Engineering,

Huazhong University of Science and Technology,

Wuhan 430074, China

$(\bowtie)$ e-mail: yuanxm@mail.hust.edu.cn
2050, respectively. China wind power generation will meet $17 \%$ of its electricity consumption by 2050 . By the end of 2010 , the total worldwide wind power installation reached $170 \mathrm{GW}$, with $44 \mathrm{GW}$ for China exceeding $41 \mathrm{GW}$ of the US as the largest installation by a country in the world.

With wind generation technology maturing and the cost decreasing close to grid parity, grid integration of wind on a large scale became a principal barrier for further deployment and has been receiving intensive attention across academia, industry, and government sectors. "Wind Monitoring 2011" [3] from China State Electricity Regulatory Commission (SERC) says that while utilization time of wind was 2,047 h in the year of 2010, it was $1,252 \mathrm{~h}$ for the first 6 months in 2011. Over Jan. to Aug. in 2011 in China, there were 193 wind farm tripping events, including 12 events individually losing power $>500 \mathrm{MW}$. In Europe and the US, however, wind utilization was a more favorable situation due to close to load center deployment of wind and the grid interfacing performance of the turbine. In spite of this, it is expected that integration issues will aggravate with upcoming large-scale development in remote rich wind areas, such as the great plains area in the US and offshore wind areas in Europe [4].

This paper reviews differences between wind and conventional generations, provides an outline over the scope of grid integration problems and the inherent associations, and discusses thoughts toward potential solutions and relevant areas to be researched.

\section{Differences between wind and conventional power generations}

(1) In terms of primary fuel and static characteristics of output power, conventional generation relying on coal 
or gas is constant and deterministic, while wind generation relying on wind is fluctuating and stochastic.

(2) In terms of equipment control and dynamic characteristics of output power, conventional generation is designed to mitigate and ride through grid disturbances, while wind generation is mostly designed not to mitigate grid disturbances and sometimes not to ride through grid disturbances.

\section{Basic problems in large-scale wind integration}

\subsection{Supply adequacy}

The fluctuating and stochastic nature of wind power imposes new challenges to supply adequacy in electric power systems [5, 6]. Load fluctuation or generation failure also causes a supply adequacy problem in conventional power systems. Based on static characteristics of load, the process of secure supply adequacy involves load forecasting, generation and grid planning, generation dispatch, etc. Planned generation usually consists of three types: on-line fast response generations (minutes) for frequency regulation, fast response generations such as hydro or gas for load following (hour), and slow response generations such as coal or nuclear for unit commitment (day). With large-scale wind generation in the system, the net load of the system exhibits two new features: increasing rate and range of variation; and increasing uncertainty of rate and range of variation. The study shows that these features will first impact load following requirements (increase), further on base load requirements (decrease) when the penetration grows, and yet at load level and on-off operation of conventional generations. Large-scale wind integration therefore requires a substantial increase for flexibility of response from conventional generations [1].

\subsection{Operation stability}

The inability of wind turbines to mitigate or ride through grid disturbances poses new challenges to operation stability in electric power systems [7]. Small signal or large signal disturbances also cause stability problems in conventional electric power systems. Based on dynamic characteristics of the load, the process to secure operation stability involves disturbance identification, dynamic control of generations, special protection schemes for system security, etc. Dynamic control of generations responds to disturbances in three ways: active power responses to mitigate phase disturbance, active power responses to mitigate frequency disturbance, and reactive power responses to mitigate voltage disturbance. With large-scale wind generation in the system, the net dynamics of generations exhibits two new features: decreasing ability to mitigate disturbances and decreasing ability to survive disturbances. The study shows that these features first impact voltage stability at the interconnection and further impact angle and frequency stability of the system when the penetration level grows. Large-scale integration of wind therefore requires a substantial increase of the ability of wind plants to mitigate and survive grid disturbances.

\section{Relationships between supply adequacy and operation stability}

Supply adequacy is a problem of static characteristics of wind power output determined from wind as primary fuel, in the time scale of minutes or above, while operation stability is a problem of dynamic characteristics of wind power output determined from turbine controls, in the time scale of seconds or below. Supply adequacy will be reached by regulating static power output with an objective of real time balancing of static power output, while operation stability will be reached by controlling dynamic power output with an objective of real time balancing of dynamic power output. The static power output characteristics of wind determine power flow and operation point of the system and therefore impact the dynamics of generations and stability of operation; on the other hand, the dynamics of wind power output determines the stability margin of the system and therefore imposes further constraints on power flow and operation point of system, as shown in Fig. 1.

As a result, turbine control is the primary factor that determines operation stability, while the fluctuation of wind power is the secondary factor for operation stability. Meanwhile, the fluctuation of wind power is the primary factor that determines supply adequacy, while turbine control is the secondary factor for supply adequacy. The system shows the operation stability challenges even with

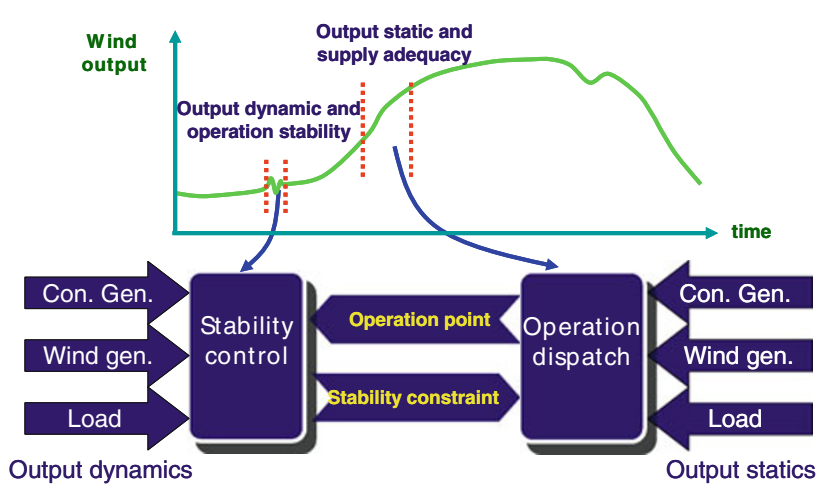

Fig. 1 Relationship between supply adequacy and operation stability 
constant wind power output and shows the supply adequacy challenges even with a synchronous generator connecting wind to the grid.

\section{Conceptual solutions to supply adequacy and operation stability problems}

Fundamental solutions for large-scale integration of wind are as follows:

(1) increasing the flexibility of conventional generations in responding to wind fluctuation to improve the compatibility of the grid to wind;

(2) optimizing disturbances to improve the compatibility of wind to the grid.

Areas around supply adequacy in the following are critical as well:

(1) Forecasting and controlling wind power fluctuation and its uncertainty.

(2) Approaches for dispatching compatible with wind power fluctuation and its uncertainty.

(3) Applications of load and other dispatchable resources. Solutions to supply adequacy problem must be based on a wide area grid platform, taking into account multiple time- and spatial-scale coherences of fluctuations among wind plants and with load fluctuations, and dispatching of flexible resources over a wide area grid platform.

Meanwhile, areas around operation stability in the following are also critical:

(1) Identification and control of grid disturbances and its characteristics.

(2) Special protection schemes of a system compatible with dynamic characteristics of wind to grid disturbances.

(3) Optimization of dynamics of conventional generation to grid disturbances, and applications of other auxiliary stabilizing units.

Solutions to the operation stability problem must be based on a local wind plant platform, taking into account multiple time- and spatial-scale dynamic interactions among wind turbines, interactions with conventional generations and loads, as well as optimization of wind turbine dynamics over a local wind plant platform.

Figure 2 illustrates basic concepts for solutions to the supply adequacy and operation stability problems.

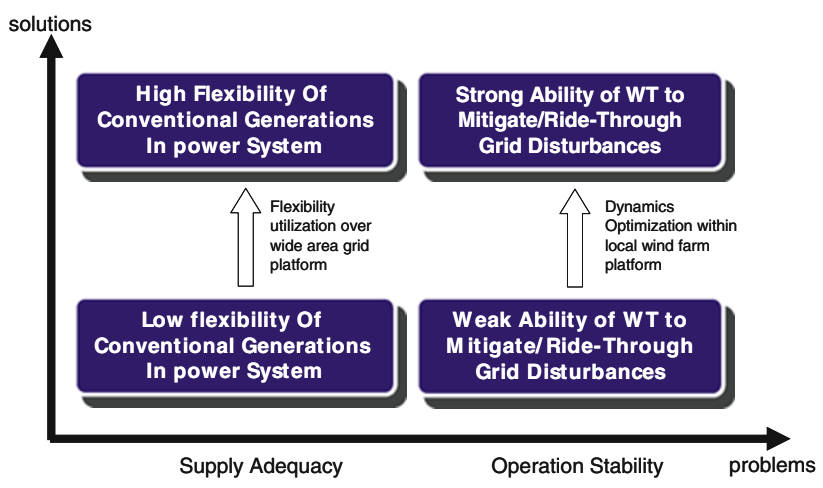

Fig. 2 Conceptual solutions for supply adequacy and operation stability problems

\section{Critical research areas for supply adequacy and operation stability}

Supply adequacy research shall start from the static characteristics of the output power as responding to atmospheric variation of a single turbine, moving to multiple turbines in a wind farm and further multiple farms in an area, finding interaction and smoothing mechanisms of turbine output power over a large geographical area, and identifying impact on reserve requirements of different time scales (flexibility) in the system. Forecasting and controlling (to the extent of available wind, rape rate control, for example) output power production in a wind farm or an area is important, providing basis for planning needs for reserve requirements of different time scales (flexibility) from conventional generations or other dispatchable resources. A dispatch strategy considering wind output forecasting uncertainty and wind output contingency is the grid-level tool insuring supply adequacy.

Meanwhile, operation stability research will start from the dynamic characteristics of output power as responding to grid electrical disturbances of a single turbine, moving to multiple turbines in a wind farm and further multiple farms in an area, finding interaction and aggregating mechanisms of turbine output power dynamics over especially long distance connection to grids, and identifying impact on grid stability of different voltage dimensions (phase angle, amplitude, and frequency) and time scales. Identifying features of grid disturbances and optimizing dynamics of wind turbines or farms are important to provide a basis for evaluating needs for optimizing dynamics of conventional generations or FACTS devices. Special protection systems considering stability emergencies are the grid-level countermeasures for insuring operation stability.

Figure 3 shows critical research topics on supply adequacy and operation stability. 


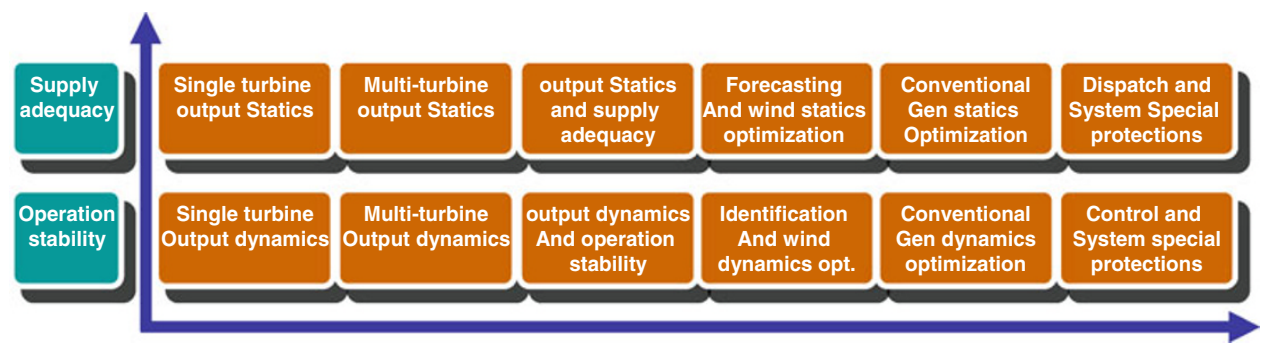

Fig. 3 Critical research topics on supply adequacy and operation stability

\section{Conclusions}

The fluctuating and stochastic characteristics of wind power output impose challenges to supply adequacy in power systems, while the weak ability of wind turbines to mitigate and ride through grid disturbances imposes challenges to operation stability in a power system. Wind power fluctuation and uncertainty determine power flow and operation point in the power system, while the grid disturbance characteristics of wind turbines impose power flow constraints and stability margins on the power system, both of which will impact limits of penetration levels. The basic solution to the supply adequacy problem is to increase flexibility in a power system in order to respond to wind fluctuations, based on a wind area grid platform, while the basic solution to operation stability is to optimize response of wind turbines to grid disturbances, based on a local wind plant platform.

Open Access This article is distributed under the terms of the Creative Commons Attribution License which permits any use, distribution, and reproduction in any medium, provided the original author(s) and the source are credited.

\section{References}

[1] $20 \%$ wind energy by 2030: increasing wind energy's contribution to US electricity supply. DOE/GO-102008-2567, National Renewable Energy Laboratory, US Department of Energy, Washington, DC, USA (2008)
[2] Zervos A, Kjaer C (2008) Pure power: wind energy scenarios up to 2030. European Wind Energy Association, Brussels

[3] Kun Yang (2011) Wind monitoring 2011. State Electricity Regulatory Commission (SERC), Beijing

[4] Wiser R, Bolinger M (2011) 2010 wind technologies market report. Lawrence Berkeley National Laboratory, Berkeley

[5] Accommodating high levels of variable generation. Accommodating high levels of variable generation. North American Electric Reliability Corporation, Princeton (2008)

[6] Denholm P, Ela E, Kirby B et al (2010) The role of energy storage with renewable electricity generation. NREL/TP-6A247187, National Renewable Energy Laboratory, US Department of Energy, Washington, DC, USA

[7] Xiaoming Yuan (2007) Integrating large wind farms into weak power grids with long transmission lines. Trans China ElectroTech Soc 22(7):29-36

\section{Author Biography}

Xiaoming YUAN Senior member, IEEE, College of Electrical and Electronic Engineering, Huazhong University of Science and Technology, Wuhan 430074, China. 